\title{
Optimal Kiloelectron Volt for Noise-Optimized Virtual Monoenergetic Images of Dual-Energy Pediatric Abdominopelvic Computed Tomography: Preliminary Results
}

Taek Min Kim, $M D^{1,2}$, Young Hun Choi, MD ${ }^{1,2}$, Jung-Eun Cheon, MD, PhD ${ }^{1,2,3}$, Woo Sun Kim, MD, PhD ${ }^{1,2,3}$, In-One Kim, MD, $\mathrm{PhD}^{1,2,3}$, Ji Eun Park, MD, $\mathrm{PhD}^{4}$, Su-mi Shin, MD, PhD ${ }^{5}$, Seong Yong Pak, BA ${ }^{6}$, Bernhard Krauss, $\mathrm{PhD}^{7}$

${ }^{1}$ Department of Radiology, Seoul National University Hospital, Seoul, Korea; ${ }^{2}$ Department of Radiology, Seoul National University College of Medicine, Seoul, Korea; ${ }^{3}$ Institute of Radiation Medicine, Seoul National University Medical Research Center, Seoul, Korea; ${ }^{4}$ Department of Radiology, Kyung Hee University Hospital, Seoul, Korea; ${ }^{5}$ Department of Radiology, Seoul Metropolitan Government-Seoul National University Boramae Medical Center, Seoul, Korea; ${ }^{6}$ Siemens Healthineers, Seoul, Korea; ${ }^{7}$ Siemens Healthineers, Forchheim, Germany

Objective: To compare quantitative and qualitative image quality parameters in pediatric abdominopelvic dual-energy CT (DECT) using noise-optimized virtual monoenergetic image (VMI) and conventional VMI at different kiloelectron volt (keV) levels.

Materials and Methods: Thirty-six consecutive abdominopelvic DECT scans were retrospectively included. Noise-optimized VMI and conventional VMI were reconstructed at seven energy levels, from $40 \mathrm{keV}$ to $100 \mathrm{keV}$ at $10 \mathrm{keV}$ intervals. The contrastto-noise ratio (CNR) and signal-to-noise ratio (SNR) of the liver, pancreas, and aorta were objectively measured and compared. Image quality was evaluated subjectively regarding image noise, image blurring of solid organ, bowel image quality and severity of beam-hardening artifacts. Optimal monoenergetic levels in keV for both algorithms were determined based on overall image quality score.

Results: The maximal CNR and SNR values for all investigated organs were observed at $40 \mathrm{keV}$ in noise-optimized VMI (CNR and SNR of liver, pancreas, aorta in order [CNR; 20.93, 17.34, 46.75: SNR; 37.39, 33.80, 63.21]), at 60-70 keV and at $70 \mathrm{keV}$ in conventional VMI (CNR; 8.12, 5.67, 15.97: SNR; 19.57, 16.66, 26.65). In qualitative image analysis, noise-optimized VMI and conventional VMI showed the best overall image quality scores at $60 \mathrm{keV}$ and at $70 \mathrm{keV}$, respectively. Noise-optimized VMI at $60 \mathrm{keV}$ showed superior CNRs, SNRs, and overall image quality scores compared to conventional VMI at $70 \mathrm{keV}(p<0.001)$. Conclusion: Optimal energy levels for noise-optimized VMI and conventional VMI were $60 \mathrm{keV}$ and at $70 \mathrm{keV}$, respectively. Noiseoptimized VMI shows superior CNRs, SNRs and subjective image quality over conventional VMI, at the optimal energy level. Keywords: Dual-energy pediatric abdominopelvic CT; Virtual monoenergetic image; Noise-optimized VMI; Conventional VMI; Image quality

\section{INTRODUCTION}

Dual-energy CT (DECT) is now re-emerging in the field of clinical radiology with the recent technical developments in CT (1). By using two different $\mathrm{X}$-ray energy spectra or tube voltages, DECT allows for a number of applications including

Received August 16, 2017; accepted after revision April 30, 2018.

Corresponding author: Young Hun Choi, MD, Department of Radiology, Seoul National University Hospital, 101 Daehak-ro, Jongno-gu, Seoul 03080, Korea.

- Tel: (822) 2072-3608•Fax: (822) 747-5781•E-mail: iater@snu.ac.kr

This is an Open Access article distributed under the terms of the Creative Commons Attribution Non-Commercial License (https:// creativecommons.org/licenses/by-nc/4.0) which permits unrestricted non-commercial use, distribution, and reproduction in any medium, provided the original work is properly cited. 
monoenergetic extrapolation. Virtual monoenergetic image (VMI) reconstruction at several different energies (kiloelectron volts [keVs]) is possible by performing a postprocessing algorithm, revealing various image parameters such as attenuation and noise from a single CT exposure (2). The benefit of this post-processing algorithm is well established for improving iodine contrast in low keV CT angiography images and reducing metal artifacts in high keV CT angiography images (3-5). However, quantitative image quality, such as contrast-to-noise ratio (CNR) or signal-tonoise ratio (SNR), remain reduced in low keV levels given that the increase in image noise at low keV levels is greater than the increase in contrast attenuation $(2,6)$.

Recently, an noise-optimized VMI was introduced that performs a regional spatial frequency-based recombination of higher signals at lower energies and superior noise properties at medium energies, to avoid increased noise at lower calculated energies (7). Objective and subjective image quality at low keV levels (40-60 keV) has been shown superior compared to that of the conventional technique in adult studies (8-10).

However, DECT imaging for children can be actually different from adults. In technical aspect, dual-source DECT technique is particularly useful and advocated in children more than other dual-energy techniques due to its better radiation dose efficiency and the maximized dual energy spectral contrast, which can be achievable in children using a newer generation of dual-source CT system (1). Regarding the optimal keV level of VMI, it would be different from adults. Because of the small body diameters of children, the transmitted $X$-ray spectrum is much softer than for adults. Hence, the optimal energy level of the virtual monochromatic imaging could be lower than for adults. Additionally, reasonably higher image quality is required for children due to their lower proportion of body fat and smaller size than adults, which may lead to subjectively different optimal VMI energy level.

To the best of our knowledge, the optimal energy level, using noise-optimized or conventional algorithm in dualsource dual-energy abdominopelvic CT in pediatrics, has not been investigated. Thus, the purpose of this study was to determine the optimal VMI energy levels with both conventional and noise-optimized monoenergetic reconstruction algorithms in order to maximize image quality in abdominopelvic DECT in pediatric patients and to compare image quality between two monoenergetic reconstruction algorithms.

\section{MATERIALS AND METHODS}

This retrospective, single-center study was approved by our Institutional Review Board. The requirement for written informed consent was waived due to the mandatory nature of abdominopelvic CT examination in routine clinical practice.

\section{Patient Selection}

We evaluated a total of 37 abdominopelvic DECT images of pediatric patients (aged younger than 18 years), performed at our institution from July to November 2015, using a standard DECT protocol for the following indications: abdominal pain $(n=12)$, fever $(n=1)$, evaluation for hidden malignancy $(n=1)$, routine follow-up for lymphoma $(n=12)$, neuroblastoma $(n=4)$, rhabdomyosarcoma $(n=2)$, Wilm's tumor $(n=2)$, hepatoblastoma $(n=1)$, immature teratoma $(n=1)$, mixed germ cell tumor $(n=$ $1)$, and rhabdoid tumor of the kidney $(n=1)$. Because dose-neutrality of dual-source DECT has been validated in previous studies $(11,12)$ and it offers additional advantages of post-processing images such as virtual noncontrast image, iodine map that are unavailable with single energy $\mathrm{CT}$, routine abdominopelvic $\mathrm{CT}$ was performed with dual-source DECT technique in that period according to our department policy.

One patient with severe pancreatic atrophy was excluded due to difficulty in measuring the region of interest (ROI) in the pancreas. Thus, 36 consecutive abdominopelvic DECT examinations acquired from 36 children (mean age, 9.7 years; range, 13 months-17 years; 27 boys and 9 girls), were included in our study.

\section{Image Acquisition}

Imaging data was acquired with a 128-channel scanner (SOMATOM Definition Flash; Siemens Healthineers, Forchheim, Germany) using two X-ray tubes with two different $\mathrm{kV}$ settings, $80 \mathrm{kVp}$ and $140 \mathrm{kVp}$. Detailed settings were as follows: detector collimation, $128 \times 0.6 \mathrm{~mm}$; rotation time, 0.28 seconds; pitch, 0.6 ; reference tube current-time product for $80 \mathrm{kVp}$ tube, $200 \mathrm{mAs}$ and for Sn140 kVp tube, $77 \mathrm{mAs}$. Scan range included from top of liver to pubic symphysis. A total amount of $1.8 \mathrm{~mL} / \mathrm{kg}$ of iodinated contrast media at a concentration of $350 \mathrm{mgI} /$ $\mathrm{mL}$ (iobitridol, Xenetix 350; Guerbet, Aulnay-sous-Bois, France) was administered via a peripheral vein of the upper extremity for 50 seconds, followed by a saline chase up 
to $20 \mathrm{~mL}$ using an automatic power injector (Envision CT; Medrad, Pittsburgh, PA, USA). DECT images were acquired at a fixed delay of 80 seconds after initiation of contrast administration. Intravenous saline injection was stopped at the beginning of the CT scanning. In our institution, the late portal-venous phase at 80 seconds after contrast media administration was chosen for DECT, because homogeneous enhancement of hepatic vessels, hepatic parenchyma, and renal parenchyma including the medulla can be achieved in the late portal-venous phase. Although a fixed delay technique may not be widely used in children, we decided to apply a fixed-delay time, because it is technically simple and it would suffice for most routine indications in children (13). Seven children (19.44\%) were sedated with per-oral chloral hydrate syrup $(50 \mathrm{mg} / \mathrm{kg})$.

Monoenergetic image sets were reconstructed from 40 $\mathrm{keV}$ to $100 \mathrm{keV}$ at $10 \mathrm{keV}$ intervals using both conventional and noise-optimized reconstruction algorithms, resulting in 7 image sets for each algorithm. Although DECT, as used for this study, enables reconstruction of monoenergetic images above $100 \mathrm{keV}$, we did not perform reconstruction at higher keV levels, because iodine attenuation is likely to be too faint (7). All image series were reconstructed with dedicated iterative reconstruction (SAFIRE; Siemens Healthineers) using a strength level of 3 and a mediumsmooth soft-tissue DECT kernel (Q30f).

\section{Quantitative Image Analysis}

The 36 DECT examinations that were reconstructed resulted in a total of 504 image sets. For every VMI image set, one radiologist ( 3 years of pediatric imaging experience) obtained a mean and standard deviation (SD) CT attenuation, in Hounsfield units (HUs), for each organ, by manually placing round ROIs at the same image level. The reader was blinded to the reconstruction sets, and used a commercially available picture archiving and communications system workstation (Infinitt PACS; Infinitt Healthcare, Seoul, Korea) on a standard reading monitor (ME315L; Totoku Electric Co. Ltd., Tokyo, Japan).

The attenuation and SD of the aorta were obtained from a single drawn ROI (mean area, $61 \mathrm{~mm}^{2}$; range, 19-150 $\mathrm{mm}^{2}$ ) as large as the lumen of the vessel. The attenuation and SD of the liver were obtained as the mean of four ROIs (mean area, $166 \mathrm{~mm}^{2}$; range, $82-288 \mathrm{~mm}^{2}$ ) positioned in anterior-posterior segments of the right hepatic lobe and medial-lateral segments of the left hepatic lobe. Large hepatic vessels and focal lesions in liver parenchyma were carefully avoided. The attenuation and SD of the pancreas were obtained as the mean of three ROIs (mean area, 65 $\mathrm{mm}^{2}$; range, 23-124 $\mathrm{mm}^{2}$ ), which were positioned the level of the pancreatic head, body, and tail. Large vessels, pancreatic ducts, and focal lesions in the pancreatic parenchyma were carefully avoided. The attenuation and SD of the paraspinal muscle was obtained from a single ovoid ROI (mean area, $117 \mathrm{~mm}^{2}$; range, 23-336 $\mathrm{mm}^{2}$ ) with avoiding areas of fat infiltration on the image obtained at the level of the iliac crest. The attenuation and SD of the subcutaneous fat of anterior abdominal wall was obtained from a single ovoid ROI (mean area, $86 \mathrm{~mm}^{2}$; range, 6-280 $\mathrm{mm}^{2}$ ). We obtained the attenuation and SD of the air in the bowel lumen by drawing a round ROI (mean area, $128 \mathrm{~mm}^{2}$; range, $64-235 \mathrm{~mm}^{2}$ ). Image noise was defined as the SD of the pixel values obtained from air (14).

All measurements were carried out in triplicate and averaged to ensure accuracy of the values. Image sets from the same examination, including size, shape, and position of the ROIs, were kept constant by applying a copy and paste function at the workstation (Fig. 1).

For each of the image sets, the CNR and SNR, relative to the muscle for the aorta, liver, and pancreas were calculated using the following equations: $\mathrm{CNR}=(\mathrm{ROIO}-\mathrm{ROIm}) / \mathrm{SDn}$, SNR = ROIo / SDn, where ROIo is the mean attenuation for the organ of interest, ROIm is the mean attenuation for the paraspinal muscles, and SDn is the mean image noise (15).

\section{Qualitative Image Analysis}

Subjective image quality was assessed by two radiologists

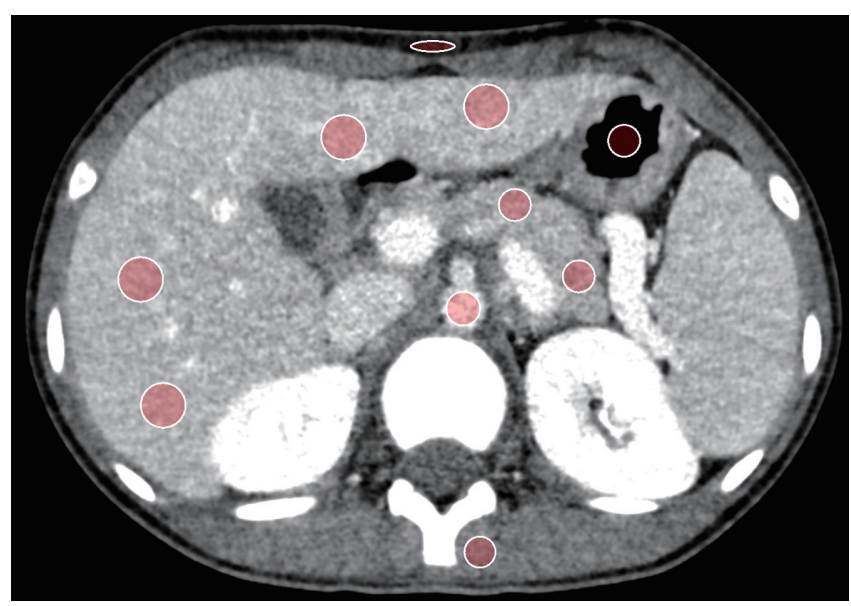

Fig. 1. Manually drawn regions of interest in liver, pancreas, aorta, paraspinal muscle, subcutaneous fat of anterior abdominal wall, and air column. All measurements were kept constant across VMI levels by using copy-and-paste function at workstation. VMI = virtual monoenergetic image 
(with 12 and 3 years of pediatric imaging experience, respectively). The reviewers were blinded to the applied reconstruction technique and VMI energy level.

Using a 5-point Likert scale, image noise (ranging from $1=$ extensive image noise to $5=$ absence of noise) and image blurring (ranging from 1 = severe blurring, edge definition very poor, margins difficult to discern to $5=$ no blurring, edges well defined, margins crisp) of solid organs were respectively scored. Bowel image quality $(1=$ bad, no diagnosis possible; 2 = poor, diagnostic confidence substantially reduced; $3=$ moderate, but sufficient for diagnosis; 4 = good; 5 = excellent) was respectively evaluated. Additionally, severity of beam hardening artifact (ranging from $1=$ massive streak artifacts that obscure the boundaries of the affected anatomic structure to $5=$ barely perceptible) was also rated (16). Overall image quality was scored by averaging the each value.

\section{Radiation Dose Evaluation}

Volume CT dose index (CTDIvol) and dose length product (DLP) were obtained by reviewing the dose reports from each examination. CTDIvol was referenced to a $32-\mathrm{cm}$ phantom.

\section{Statistical Analysis}

Paired $t$ test was used for comparing quantitative and qualitative image parameters between noise-optimized VMI and conventional VMI in each same energy level. Significant differences in mean attenuation, noise, SNR and CNR between different virtual monoenergetic keV-levels, for the liver, pancreas, and aorta, were evaluated using repeatedmeasures analysis of variance. Post hoc analysis was conducted using the Dunnett test. Optimal monoenergetic levels (keV) for noise-optimized VMI and conventional VMI were determined based on overall image quality scores. Improvement ratios of noise-optimized VMI compared to conventional VMI at optimal keV levels were calculated for mean attenuation, noise, SNR, and CNR. Weighted Cohen's kappa coefficients ( $\kappa$-values) were calculated for the assessment of interobserver agreements of overall image quality, bowel image quality, image noise and severity of beam hardening artifact. Kappa values were interpreted as follows: absence of agreement $\leq 0$, poor agreement $<0.20$, fair agreement $0.21-0.40$, moderate agreement $0.41-0.60$, good agreement $0.61-0.80$ and excellent agreement $>0.80$.

We classify all patients into two groups according to the patients' age, with infant or child (group 1; age $\leq 10$ years, $\mathrm{n}=20$ ) and adolescence (group 2; age $>10$ years, $\mathrm{n}=16$ ).
Subgroup analysis was performed for each group, using same methods as aforementioned.

Commercially available software packages (SPSS 21.0 for Windows; IBM Corp., Armonk, NY, USA) were used for statistical analyses. A $p$ value $<0.05$ was considered to indicate statistical significance.

\section{RESULTS}

\section{Mean Attenuation}

Mean attenuations of the liver, pancreas, aorta, and air decreased stepwise with increased keV levels, while mean attenuation of subcutaneous fat gradually increased (Fig. 2). In case of the liver, pancreas, aorta and subcutaneous fat, noise-optimized VMI showed significantly higher attenuation than conventional VMI in the liver at 40-60 $\mathrm{keV}$, pancreas at $40-50 \mathrm{keV}$, subcutaneous fat at $80 \mathrm{keV}$ (all $p<0.05$ ); however, the absolute attenuation difference between the two image sets was less than $1.4 \mathrm{HU}$. In case of air, noise-optimized VMI showed significantly lower attenuation at $40-70 \mathrm{keV}$, and higher attenuation at $80-$ $100 \mathrm{keV}$ (all $p<0.05$ ). The absolute attenuation difference between the two image sets was less than $23.3 \mathrm{HU}$. At the other energy levels, there was no statistical difference in attenuation between two image sets (Table 1).

\section{Image Noise}

The image noise was lowest at $70 \mathrm{keV}$ for both noiseoptimized and conventional VMIs (Fig. 3). With noiseoptimized monoenergetic algorithm, noise decreased from $7.21 \pm 1.31$ (mean \pm SD) at $40 \mathrm{keV}$ to $6.44 \pm 0.81$ at $70 \mathrm{keV}$.

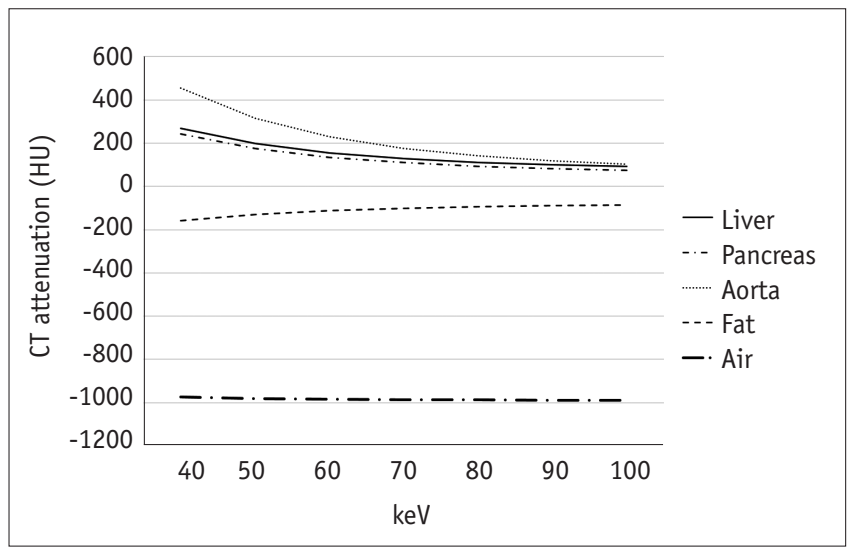

Fig. 2. Graphs of mean attenuation of liver, pancreas, aorta, subcutaneous fat, and air in mean of noise-optimized and conventional algorithms. $\mathrm{HU}=$ Hounsfield unit, $\mathrm{keV}=$ kiloelectron volt 
Table 1. Attenuation Number (HU) of Three Measured Organs, Subcutaneous Fat, and Air in Series Reconstructed with NoiseOptimized and Conventional Algorithms (keV)

\begin{tabular}{|c|c|c|c|c|c|c|c|}
\hline & $40 \mathrm{keV}$ & $50 \mathrm{keV}$ & $60 \mathrm{keV}$ & $70 \mathrm{keV}$ & $80 \mathrm{keV}$ & $90 \mathrm{keV}$ & $100 \mathrm{keV}$ \\
\hline \multicolumn{8}{|l|}{ Liver } \\
\hline $\begin{array}{l}\text { Noise-optimized } \\
\text { VMI }\end{array}$ & $269.61 \pm 46.52$ & $198.65 \pm 31.07$ & $155.53 \pm 21.74$ & $128.90 \pm 16.41$ & $111.76 \pm 13.25$ & $100.51 \pm 11.32$ & $92.08 \pm 10.59$ \\
\hline Conventional VMI & $268.49 \pm 46.16$ & $197.90 \pm 30.71$ & $155.26 \pm 21.75$ & $128.98 \pm 16.57$ & $112.06 \pm 13.39$ & $100.23 \pm 11.66$ & $93.17 \pm 10.31$ \\
\hline$p$ value & $0.008^{*}$ & $<0.001^{*}$ & $0.009^{*}$ & 0.212 & $0.005^{*}$ & 0.688 & 0.076 \\
\hline \multicolumn{8}{|l|}{ Pancreas } \\
\hline $\begin{array}{l}\text { Noise-optimized } \\
\text { VMI }\end{array}$ & $243.67 \pm 37.94$ & $176.11 \pm 24.61$ & $134.86 \pm 17.13$ & $109.54 \pm 12.62$ & $93.65 \pm 9.22$ & $83.04 \pm 7.30$ & $75.63 \pm 6.30$ \\
\hline Conventional VMI & $241.57 \pm 37.02$ & $175.46 \pm 24.00$ & $134.91 \pm 16.71$ & $109.77 \pm 12.07$ & $93.77 \pm 9.38$ & $83.03 \pm 7.58$ & $75.65 \pm 6.77$ \\
\hline$p$ value & $0.003^{*}$ & $0.041^{*}$ & 0.862 & 0.454 & 0.347 & 0.951 & 0.937 \\
\hline \multicolumn{8}{|l|}{ Aorta } \\
\hline $\begin{array}{l}\text { Noise-optimized } \\
\text { VMI }\end{array}$ & $455.78 \pm 57.66$ & $314.58 \pm 38.41$ & $228.74 \pm 27.51$ & $175.87 \pm 20.79$ & $142.09 \pm 16.64$ & $119.53 \pm 14.67$ & $103.49 \pm 13.44$ \\
\hline Conventional VMI & $449.81 \pm 66.78$ & $313.75 \pm 38.12$ & $228.19 \pm 26.89$ & $175.64 \pm 20.81$ & $141.96 \pm 17.14$ & $119.48 \pm 15.27$ & $103.80 \pm 14.10$ \\
\hline$p$ value & 0.261 & 0.186 & 0.212 & 0.219 & 0.630 & 0.761 & 0.580 \\
\hline \multicolumn{8}{|l|}{ Fat } \\
\hline $\begin{array}{l}\text { Noise-optimized } \\
\text { VMI }\end{array}$ & $-159.25 \pm 18.85$ & $-130.65 \pm 10.98$ & $-112.24 \pm 8.16$ & $-101.29 \pm 5.67$ & $-93.53 \pm 5.20$ & $-89.52 \pm 5.30$ & $-85.90 \pm 5.09$ \\
\hline Conventional VMI & $-160.90 \pm 18.10$ & $-130.26 \pm 11.92$ & $-112.63 \pm 7.79$ & $-101.47 \pm 5.74$ & $-94.88 \pm 4.84$ & $-89.58 \pm 4.83$ & $-86.85 \pm 4.70$ \\
\hline$p$ value & 0.120 & 0.666 & 0.643 & 0.483 & $0.014^{*}$ & 0.929 & 0.296 \\
\hline \multicolumn{8}{|l|}{ Air } \\
\hline $\begin{array}{l}\text { Noise-optimized } \\
\text { VMI }\end{array}$ & $-985.97 \pm 6.27$ & $-986.14 \pm 6.16$ & $-986.28 \pm 6.22$ & $-986.45 \pm 6.12$ & $-986.30 \pm 6.22$ & $-986.37 \pm 6.26$ & $-986.08 \pm 6.33$ \\
\hline Conventional VMI & $-962.63 \pm 15.12$ & $-975.96 \pm 9.03$ & $-982.34 \pm 5.64$ & $-985.89 \pm 6.24$ & $-988.17 \pm 7.70$ & $-989.40 \pm 8.85$ & $-990.47 \pm 9.63$ \\
\hline$p$ value & $0.003^{*}$ & $0.014^{*}$ & $0.017^{*}$ & $0.005^{*}$ & $0.042^{*}$ & $0.045^{*}$ & $0.023^{*}$ \\
\hline
\end{tabular}

Values are expressed as mean \pm SD (HU). $p$ values refer to comparison between noise-optimized and conventional VMIs. ${ }^{*}$ Statistical significance. $\mathrm{HU}=$ Hounsfield unit, $\mathrm{keV}=$ kiloelectron volt, $\mathrm{SD}=$ standard deviation, $\mathrm{VMI}=$ virtual monoenergetic image

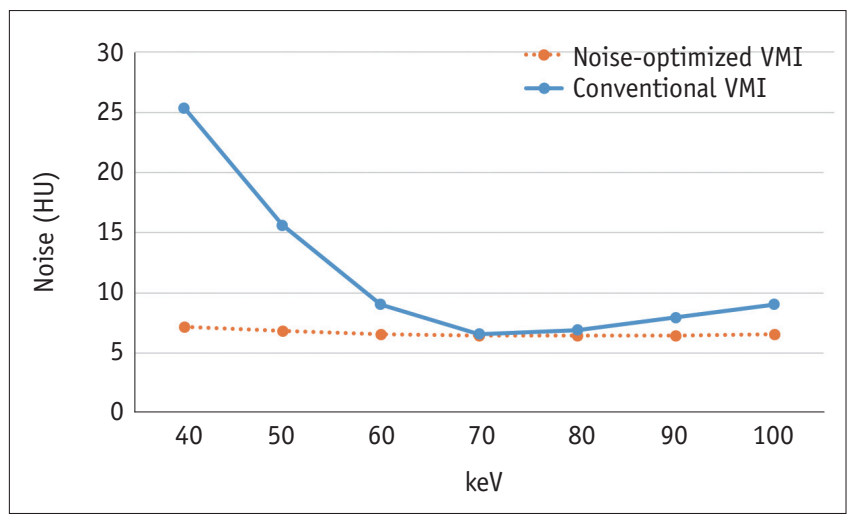

Fig. 3. Graphs of image noise in noise-optimized and conventional algorithms.

With conventional monoenergetic algorithm, mean noise decreased from $25.37 \pm 5.77$ at $40 \mathrm{keV}$ to $6.59 \pm 0.85$ at $70 \mathrm{keV}$. Image noise increased stepwise beyond the optimal energy levels for both algorithms; however, the extent of increase in noise at the higher energy levels was relatively small compared to at the lower energy levels. At all energy levels, noise-optimized VMI showed significantly lower image noise than conventional VMI (all $p<0.05$ ), except at $80 \mathrm{keV}$ (noise-optimized VMI vs. conventional VMI; $6.45 \pm$ 0.85 vs. $6.90 \pm 1.17, p=0.071$ ) (Table 2 ).

Standard deviation of subcutaneous fat was lowest at 90 $\mathrm{keV}$ and $70 \mathrm{keV}$ for noise-optimized and conventional VMIs. The values decreased from $21.76 \pm 4.42$ (mean \pm SD) at 40 $\mathrm{keV}$ to $9.01 \pm 1.83$ at $90 \mathrm{keV}$ with noise-optimized VMI, and $38.19 \pm 8.03$ at $40 \mathrm{keV}$ to $10.51 \pm 2.21$ at $70 \mathrm{keV}$ with conventional VMI.

\section{Signal-To-Noise Ratio}

Figure 4 demonstrates an overview of the calculated SNR values. The maximum SNR with noise-optimized monoenergetic algorithm was achieved at $40 \mathrm{keV}$ in all organs. SNRs in the liver, pancreas, aorta were $37.39 \pm 6.45$, $33.80 \pm 5.26$, and $63.21 \pm 8.00$, respectively. SNR peaked at $40 \mathrm{keV}$ and gradually decreased when it got to higher keV 
levels. SNR values at $40 \mathrm{keV}$ were significantly greater than those at all other energy levels in all organs $(p<0.001)$.

The maximum SNR with conventional monoenergetic algorithm was achieved at $70 \mathrm{keV}$ in all organs. SNRs in the liver, pancreas and aorta were $19.57 \pm 2.51,16.66 \pm 1.83$, and $26.65 \pm 3.16$, respectively. SNRs gradually increased at low keV levels, peaked at $70 \mathrm{keV}$, and decreased at high $\mathrm{keV}$ levels, in all organs. SNRs at $70 \mathrm{keV}$ were significantly

Table 2. Noise of Air and Subcutaneous Fat of Anterior Abdominal Wall in Series Reconstructed with Noise-0ptimized and Conventional Algorithms (keV)

\begin{tabular}{lccccccc}
\hline & $40 \mathrm{keV}$ & $50 \mathrm{keV}$ & $60 \mathrm{keV}$ & $70 \mathrm{keV}$ & $80 \mathrm{keV}$ & $90 \mathrm{keV}$ & $100 \mathrm{keV}$ \\
\hline Air & & & & & & \\
$\quad$ Noise-optimized VMI & $7.21 \pm 1.31$ & $6.82 \pm 0.95$ & $6.59 \pm 0.76$ & $6.44 \pm 0.81$ & $6.45 \pm 0.85$ & $6.46 \pm 0.84$ & $6.56 \pm 0.95$ \\
$\quad$ Conventional VMI & $25.37 \pm 5.77$ & $15.61 \pm 3.36$ & $9.06 \pm 1.70$ & $6.59 \pm 0.85$ & $6.90 \pm 1.17$ & $7.95 \pm 1.52$ & $9.04 \pm 1.67$ \\
$\quad p$ value & $<0.001^{*}$ & $<0.001^{*}$ & $<0.001^{*}$ & $0.031^{*}$ & 0.071 & $0.001^{*}$ & $<0.001^{*}$ \\
Subcutaneous fat & & & & & & \\
$\quad$ Noise-optimized VMI & $21.76 \pm 4.42$ & $15.58 \pm 3.12$ & $12.36 \pm 2.58$ & $10.35 \pm 2.11$ & $9.28 \pm 1.96$ & $9.01 \pm 1.83$ & $9.21 \pm 1.78$ \\
Conventional VMI & $38.19 \pm 8.03$ & $22.18 \pm 4.88$ & $13.67 \pm 2.94$ & $10.51 \pm 2.21$ & $10.54 \pm 1.96$ & $11.36 \pm 1.90$ & $12.31 \pm 2.14$ \\
$p$ value & $<0.001^{*}$ & $<0.001^{*}$ & $<0.001^{*}$ & 0.280 & $<0.001^{*}$ & $<0.001^{*}$ & $<0.001^{*}$ \\
\hline
\end{tabular}

Values are expressed as mean \pm SD (HU). $p$ values refer to comparison between noise-optimized and conventional VMIs. *Statistical significance.

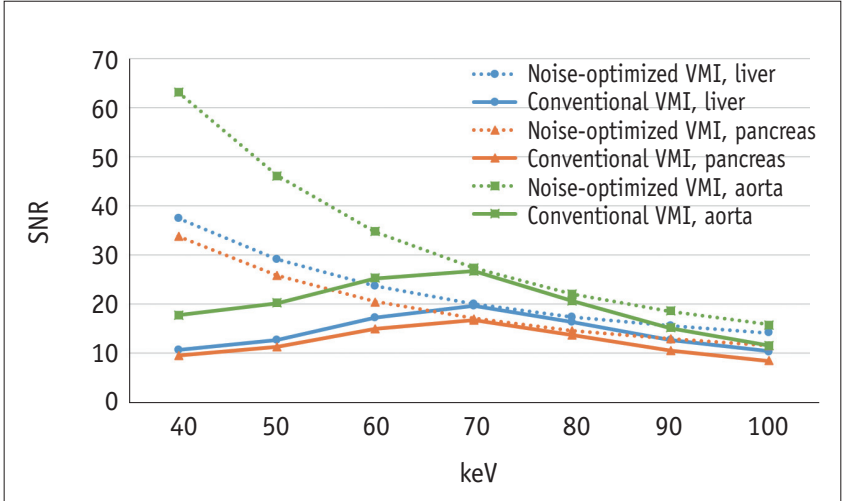

Fig. 4. Graphs showing SNR values of liver, pancreas, and aorta in noise-optimized and conventional VMIs. SNR was significantly higher in noise-optimized algorithm compared to conventional algorithm at all VMI energy levels. SNR = signal-to-noise ratio

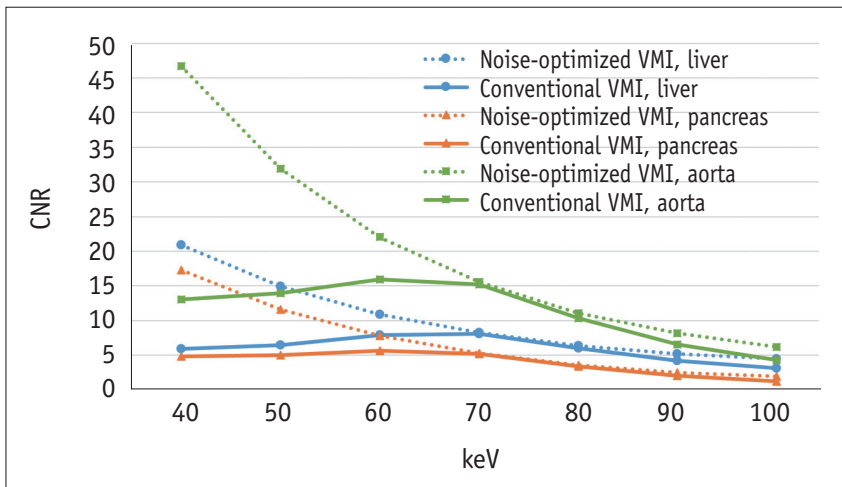

Fig. 5. Graphs of CNR values of liver, pancreas, and aorta in noise-optimized and conventional VMIs. CNR was significantly higher in noise-optimized algorithm compared to conventional algorithm in all VMI energy levels, except for $70 \mathrm{keV}$. CNR = contrastto-noise ratio

Table 3. SNR of Three Measured Organs in Series Reconstructed with Noise-Optimized and Conventional Algorithms (keV)

\begin{tabular}{|c|c|c|c|c|c|c|c|}
\hline & $40 \mathrm{keV}$ & $50 \mathrm{keV}$ & $60 \mathrm{keV}$ & $70 \mathrm{keV}$ & $80 \mathrm{keV}$ & $90 \mathrm{keV}$ & $100 \mathrm{keV}$ \\
\hline \multicolumn{8}{|l|}{ Liver } \\
\hline Noise-optimized VMI & $37.39 \pm 6.45$ & $29.13 \pm 4.56$ & $23.60 \pm 3.30$ & $20.02 \pm 2.55$ & $17.33 \pm 2.05$ & $15.56 \pm 1.75$ & $14.04 \pm 1.61$ \\
\hline Conventional VMI & $10.58 \pm 1.82$ & $12.68 \pm 1.97$ & $17.14 \pm 2.40$ & $19.57 \pm 2.51$ & $16.24 \pm 1.94$ & $12.61 \pm 1.47$ & $10.31 \pm 1.14$ \\
\hline$p$ value & $<0.001^{*}$ & $<0.001^{*}$ & $<0.001^{*}$ & $<0.001^{*}$ & $<0.001^{*}$ & $<0.001^{*}$ & $<0.001^{*}$ \\
\hline \multicolumn{8}{|l|}{ Pancreas } \\
\hline Noise-optimized VMI & $33.80 \pm 5.26$ & $25.82 \pm 3.61$ & $20.46 \pm 2.60$ & $17.01 \pm 1.96$ & $14.52 \pm 1.43$ & $12.85 \pm 1.13$ & $11.53 \pm 0.96$ \\
\hline Conventional VMI & $9.52 \pm 1.46$ & $11.24 \pm 1.54$ & $14.89 \pm 1.84$ & $16.66 \pm 1.83$ & $13.59 \pm 1.36$ & $10.44 \pm 0.95$ & $8.37 \pm 0.75$ \\
\hline$p$ value & $<0.001^{*}$ & $<0.001^{*}$ & $<0.001^{*}$ & $<0.001^{*}$ & $<0.001^{*}$ & $<0.001^{*}$ & $<0.001^{*}$ \\
\hline \multicolumn{8}{|l|}{ Aorta } \\
\hline Noise-optimized VMI & $63.21 \pm 8.00$ & $46.13 \pm 5.63$ & $34.71 \pm 4.17$ & $27.31 \pm 3.23$ & $22.03 \pm 2.58$ & $18.50 \pm 2.27$ & $15.78 \pm 2.05$ \\
\hline Conventional VMI & $17.73 \pm 2.63$ & $20.10 \pm 2.44$ & $25.19 \pm 2.97$ & $26.65 \pm 3.16$ & $20.57 \pm 2.48$ & $15.03 \pm 1.92$ & $11.48 \pm 1.56$ \\
\hline$p$ value & $<0.001^{*}$ & $<0.001^{*}$ & $<0.001^{*}$ & $<0.001^{*}$ & $<0.001^{*}$ & $<0.001^{*}$ & $<0.001^{*}$ \\
\hline
\end{tabular}

Values are expressed as mean \pm SD. $p$ values refer to comparison between noise-optimized and conventional VMIs. *Statistical significance. SNR = signal-to-noise ratio 
greater than those at all other energy levels $(p<0.001)$.

SNR was significantly higher in noise-optimized VMI compared to conventional VMI in all measured organs at each energy level $(p<0.001)$ (Table 3$)$.

\section{Contrast-To-Noise Ratio}

Figure 5 summarizes the calculated CNR values in noiseoptimized and conventional monoenergetic algorithms. The maximum CNR at noise-optimized VMI was achieved at $40 \mathrm{keV}$, in all organs (CNRs in the liver, pancreas and aorta were $20.93 \pm 7.08,17.34 \pm 6.10$, and $46.75 \pm 8.84$, respectively). All CNR values peaked at $40 \mathrm{keV}$ and gradually decreased as keV levels increased. CNR at $40 \mathrm{keV}$ was significantly greater than at all other energy levels in all organs $(p<0.001)$.

The maximum CNR at conventional VMI was achieved at $70 \mathrm{keV}$ in the liver $(8.12 \pm 2.64)$ and at $60 \mathrm{keV}$ in the pancreas $(5.67 \pm 2.18)$ and aorta $(15.97 \pm 3.31)$. CNR gradually increased at low keV levels, peaked at $60-70 \mathrm{keV}$, and decreased at high keV levels. Liver CNR at $70 \mathrm{keV}$ was significantly greater than at all other energy levels ( $p=$ 0.006 at $60 \mathrm{keV}$, otherwise, $p<0.001$ ). Pancreas and aorta CNR at $60 \mathrm{keV}$ were significantly greater than at all other energy levels $(p<0.001)$.

CNR was significantly higher in noise-optimized VMI compared to conventional VMI at each energy level $(p<$ 0.001 ), except for $70 \mathrm{keV}$ in pancreas (noise-optimized VMI vs. conventional VMI; $5.29 \pm 2.38$ vs. $5.21 \pm 2.23, p=0.158$ ) (Table 4).

\section{Subjective Image Analysis}

The mean values of subjective image analysis in noise- optimized and conventional VMI in all energy levels of two readers were summarized in Table 5.

In noise-optimized VMI, image noise score of solid organ (mean; 3.75 and 3.78 in reader 1 and 2) and severity score of beam-hardening artifact (3.75 and 3.67) were highest in $70 \mathrm{keV}$ in both two readers. And $60 \mathrm{keV}$ showed the highest score at image blurring of solid organ (3.94 and 3.86) and bowel image quality (3.92 and 3.69) in both two readers. As a result, despite the best CNR being achieved at $40 \mathrm{keV}$ in all measured organs, $60 \mathrm{keV}$ scored as the best overall image quality (3.72 and 3.64) in both two readers due to higher image noise, image blurring, lower bowel image quality and severe beam hardening artifact at keV levels less than $60 \mathrm{keV}$ (Fig. 6).

In conventional VMI, $70 \mathrm{keV}$ showed the highest score at image noise (3.56 and 3.44) and image blurring of solid organs (3.64 and 3.5), bowel image quality (3.5 and 3.42) and severity of beam-hardening artifact (3.61 and 3.5) in two readers (Fig. 7). Overall image quality score was also highest in $70 \mathrm{keV}$ with mean of 3.58 and 3.47 in reader 1 and 2, respectively.

Inter-reader agreement was all good in image noise $(\kappa=$ $0.71, p<0.001)$ and image blurring of solid organ $(\kappa=0.69$, $p<0.001)$, bowel image quality $(\kappa=0.65, p<0.001)$, and severity of beam-hardening artifact $(\kappa=0.75, p<0.001)$.

\section{Comparison between Noise-0ptimized VMI and}

\section{Conventional VMI in Optimal keV Levels}

The optimal keV levels for noise-optimized and conventional monoenergetic algorithms were $60 \mathrm{keV}$ and 70 $\mathrm{keV}$, respectively. Noise-optimized VMI at $60 \mathrm{keV}$ revealed significantly better SNRs, with relative difference of $20.6 \%$,

Table 4. CNR of Three Measured Organs in Series Reconstructed with Noise-0ptimized and Conventional Algorithms (keV)

\begin{tabular}{|c|c|c|c|c|c|c|c|}
\hline & $40 \mathrm{keV}$ & $50 \mathrm{keV}$ & $60 \mathrm{keV}$ & $70 \mathrm{keV}$ & $80 \mathrm{keV}$ & $90 \mathrm{keV}$ & $100 \mathrm{keV}$ \\
\hline \multicolumn{8}{|l|}{ Liver } \\
\hline Noise-optimized VMI & $20.93 \pm 7.08$ & $14.93 \pm 4.89$ & $10.92 \pm 3.51$ & $8.29 \pm 2.65$ & $6.40 \pm 2.17$ & $5.20 \pm 1.88$ & $4.45 \pm 2.19$ \\
\hline Conventional VMI & $5.93 \pm 2.00$ & $6.50 \pm 2.12$ & $7.92 \pm 2.57$ & $8.12 \pm 2.64$ & $6.04 \pm 2.04$ & $4.18 \pm 1.59$ & $3.16 \pm 1.24$ \\
\hline$p$ value & $<0.001^{*}$ & $<0.001^{*}$ & $<0.001^{*}$ & $<0.001^{*}$ & $<0.001^{*}$ & $<0.001^{*}$ & $<0.001^{*}$ \\
\hline \multicolumn{8}{|l|}{ Pancreas } \\
\hline Noise-optimized VMI & $17.34 \pm 6.10$ & $11.63 \pm 4.28$ & $7.79 \pm 3.10$ & $5.29 \pm 2.38$ & $3.59 \pm 1.83$ & $2.49 \pm 1.55$ & $1.94 \pm 1.86$ \\
\hline Conventional VMI & $4.87 \pm 1.73$ & $5.06 \pm 1.81$ & $5.67 \pm 2.18$ & $5.21 \pm 2.23$ & $3.39 \pm 1.74$ & $2.01 \pm 1.27$ & $1.22 \pm 1.02$ \\
\hline$p$ value & $<0.001^{*}$ & $<0.001^{*}$ & $<0.001^{*}$ & 0.158 & $<0.001^{*}$ & $<0.001^{*}$ & $0.008^{*}$ \\
\hline \multicolumn{8}{|l|}{ Aorta } \\
\hline Noise-optimized VMI & $46.75 \pm 8.84$ & $31.93 \pm 6.30$ & $22.03 \pm 4.64$ & $15.59 \pm 3.56$ & $11.10 \pm 2.84$ & $8.14 \pm 2.45$ & $6.19 \pm 2.47$ \\
\hline Conventional VMI & $13.08 \pm 2.87$ & $13.92 \pm 2.71$ & $15.97 \pm 3.31$ & $15.20 \pm 3.48$ & $10.37 \pm 2.74$ & $6.60 \pm 2.04$ & $4.34 \pm 1.62$ \\
\hline$p$ value & $<0.001^{*}$ & $<0.001^{*}$ & $<0.001^{*}$ & $<0.001^{*}$ & $<0.001^{*}$ & $<0.001^{*}$ & $<0.001^{*}$ \\
\hline
\end{tabular}

Values are expressed as mean \pm SD. $p$ values refer to comparison between noise-optimized and conventional VMIs. ${ }^{*}$ Statistical significance. $\mathrm{CNR}=$ contrast-to-noise ratio 


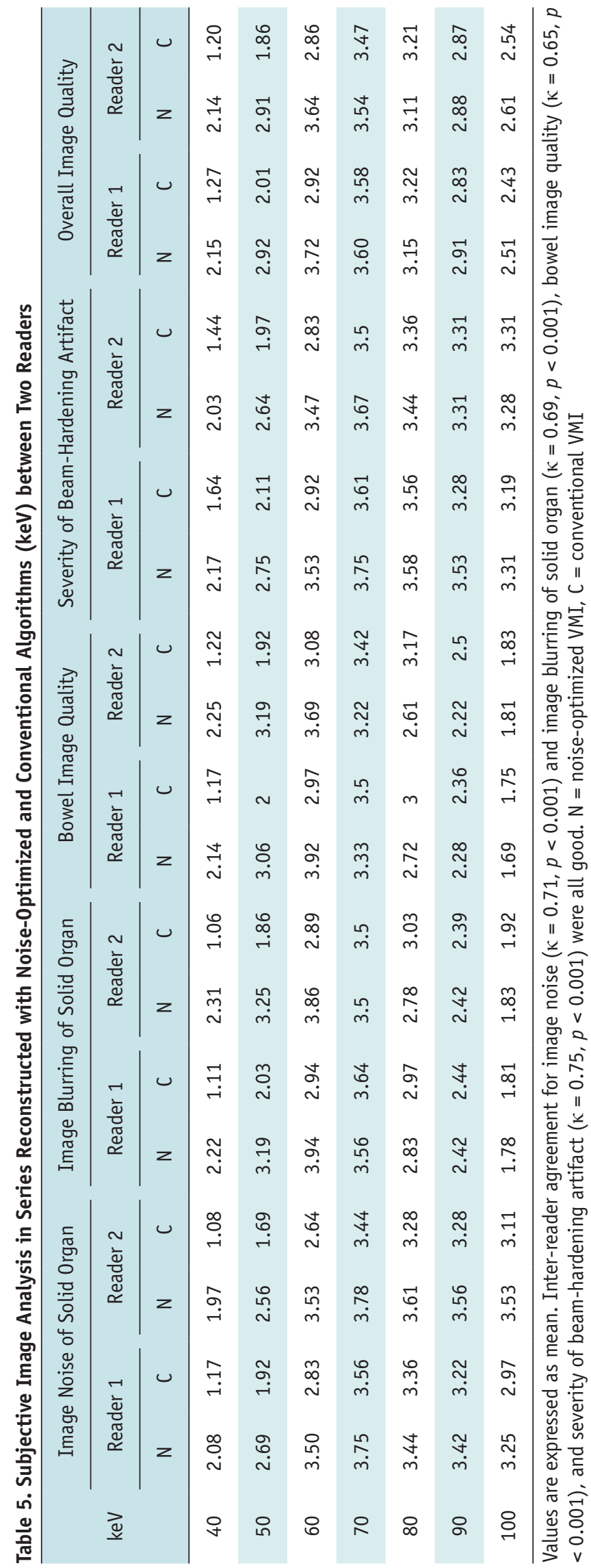

$22.9 \%$, and $30.2 \%$ (liver, pancreas, and aorta, respectively) than conventional VMI at $70 \mathrm{keV}(p<0.001)$ (Table 6). The CNR improvement of noise-optimized VMI at $60 \mathrm{keV}$ was also significant with $34.5 \%, 49.5 \%$, and $44.9 \%$ (liver, pancreas, and aorta, respectively) of relative difference compared to conventional VMI at $70 \mathrm{keV}(p<0.001)$. Image noise showed no significant difference between noiseoptimized VMI at $60 \mathrm{keV}$ and conventional VMI at $70 \mathrm{keV}$ (noise-optimized VMI vs. conventional VMI; $6.59 \pm 0.76$ vs. $6.59 \pm 0.85, p=0.982$ ).

In subjective image analysis, noise-optimized VMI at 60 $\mathrm{keV}$ received a significantly higher score than conventional VMI at $70 \mathrm{keV}$ in overall image quality in both reader 1 (noise-optimized VMI vs. conventional VMI; 3.72 vs. $3.58, p=0.048$ ) and reader 2 (noise-optimized VMI vs. conventional VMI; 3.64 vs. $3.47, p=0.042$ ).

\section{Subgroup Analysis}

The results of quantitative and qualitative image analysis for two groups were summarized in Supplementary Tables 1-5 (in the online-only Data Supplement). The optimal energy levels for maximizing CNR, SNR, minimizing image noise, scoring best overall image quality were not different between two groups, and also similar as previously shown results evaluated from total patients.

\section{Radiation Dose}

The mean CTDIvol was $3.09 \pm 1.66 \mathrm{mGy}$ (range, 0.95-5.36 $\mathrm{mGy}$ ) and the mean DLP was $134.77 \pm 91.88 \mathrm{mGy} \cdot \mathrm{cm}$ (range, 31-262 $\mathrm{mGy} \cdot \mathrm{cm}$ ) in total population.

The mean CTDIvol was $1.98 \pm 0.93 \mathrm{mGy}$ (range, $0.95-3.69$ $\mathrm{mGy}$ ) in group 1, $4.88 \pm 0.52 \mathrm{mGy}$ (range, 4-5.36) in group 2. The mean DLP was $71.5 \pm 46.95 \mathrm{mGy} \cdot \mathrm{cm}$ (range, 31-164 $\mathrm{mGy} \cdot \mathrm{cm}$ ) in group 1, $236 \pm 25.5 \mathrm{mGy} \cdot \mathrm{cm}$ (range, $20-262$ $\mathrm{mGy} \cdot \mathrm{cm})$ in group 2.

\section{DISCUSSION}

In our quantitative image analysis of abdominopelvic DECT for pediatric patients, the optimal energy levels for maximum CNR and SNR occurred at 60-70 keV and $70 \mathrm{keV,}$ respectively, in conventional algorithm and at $40 \mathrm{keV}$ in noise-optimized algorithm. In terms of subjective image quality assessment, the results were different from those of quantitative CNR and SNR analysis, especially for noiseoptimized VMI (the optimal at $60 \mathrm{keV}$ for overall image quality vs. at $40 \mathrm{keV}$ for (NR and SNR). Although CNR 

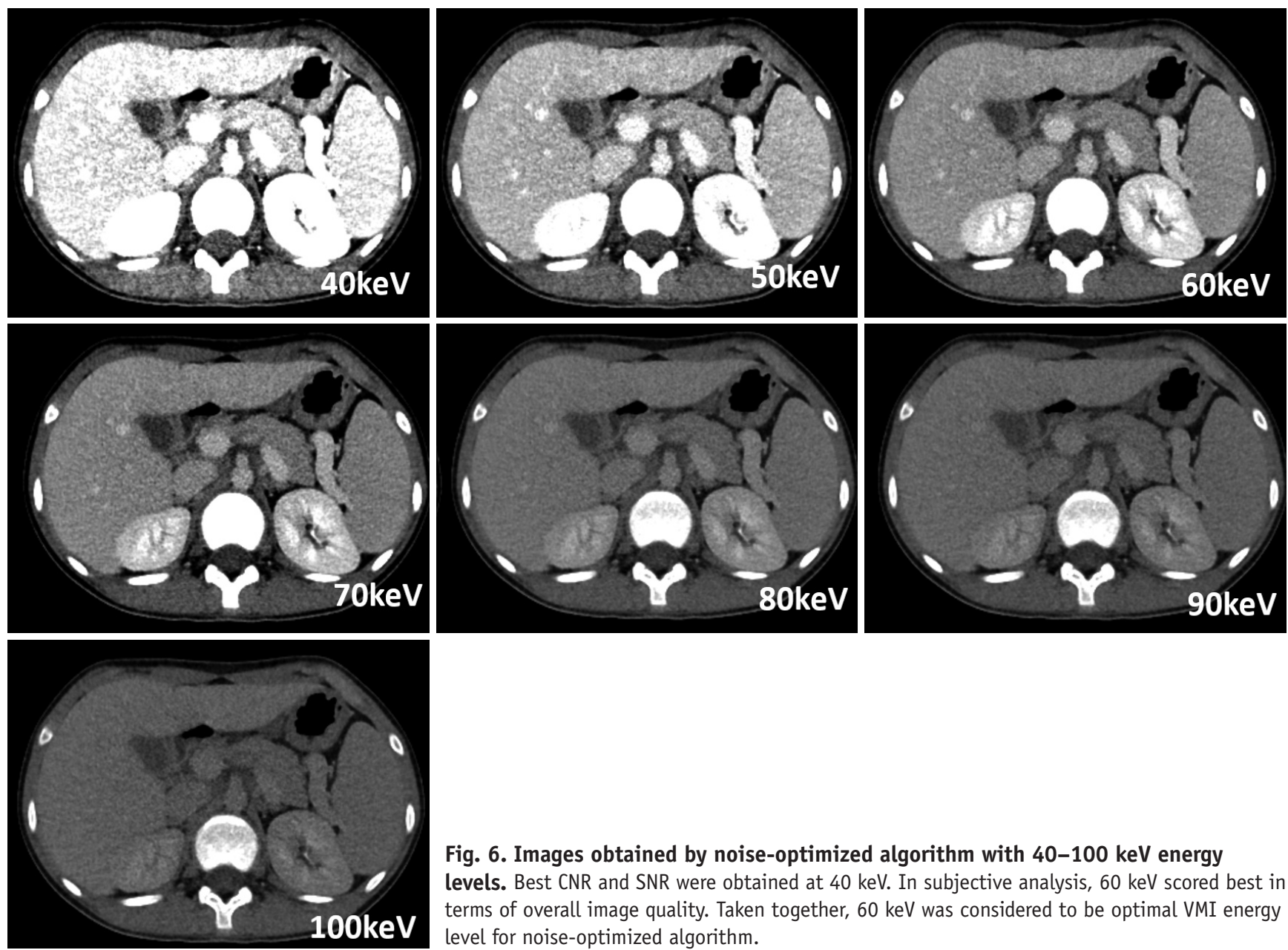

Fig. 6. Images obtained by noise-optimized algorithm with 40-100 keV energy levels. Best CNR and SNR were obtained at $40 \mathrm{keV}$. In subjective analysis, $60 \mathrm{keV}$ scored best in terms of overall image quality. Taken together, $60 \mathrm{keV}$ was considered to be optimal VMI energy level for noise-optimized algorithm.

and SNR are often used as an image quality indicator, consideration for the absolute noise level is also required. Increased CNR and SNR does not necessarily guarantee improved image quality, if the absolute noise level is clinically intolerable and a drastic increase in the absolute noise level could render an image non-diagnostic $(17,18)$. In our study, noise-optimized VMI at $40 \mathrm{keV}$ showed the highest liver CNR of 20.93 and SNR of 37.39, while the SD of the air was 7.21, and the SD of the fat was 21.76, which could be unacceptably high, when we consider the mean muscle noise level of $13.1 \mathrm{HU}$ with single-energy body CT in children $(12,19)$. On the contrary, noise-optimized VMI at $60 \mathrm{keV}$ showed the lower noise level (6.59 at air, 12.36 at fat) at the expense of loss of CNR and SNR, but still higher than those of conventional VMI at $70 \mathrm{keV}$. Subjective overall image quality score increased from 2.15 and 2.14 at $40 \mathrm{keV}$ to 3.72 and 3.64 at $60 \mathrm{keV}$ in reader 1 and 2, respectively. In this regard, we think that subjective image quality could represent overall image quality more accurately, because subjective image quality assessment incorporates all of CNR,
SNR, and noise level in addition to artifacts.

In previous adult studies, Sudarski et al. (20) reported that a conventional VMI algorithm showed optimal CNR and SNR at $60-70 \mathrm{keV}$ in dual-energy CT angiography (DE-CTA) of the abdomen. Other adult studies using dual-source DECT in other body parts have showed similar results; the optimal keV level was $70 \mathrm{keV}$ in pulmonary DE-CTA (3), $60 \mathrm{keV}$ in cerebral and cervical DE-CTA (4). In addition, DECT acquired by fast kilovoltage switching technique showed the similar results. The optimal conventional VMI energy level for highest CNR was $68 \mathrm{keV}$ in phantom study, and best image quality at $65-75 \mathrm{keV}$ in unenhanced head CT $(21,22)$. In spite of the young age of our study sample, our results are in concordance with those of previous studies, showing maximum CNR and SNR, and overall image quality score at $70 \mathrm{keV}$ with conventional VMI algorithm.

According to initial phantom study of noise-optimized VMI algorithm, the noise-optimized VMI technique provided increasing iodine CNR with decreasing keV, with the optimum CNR obtained at the lowest energy level of $40 \mathrm{keV}$ 

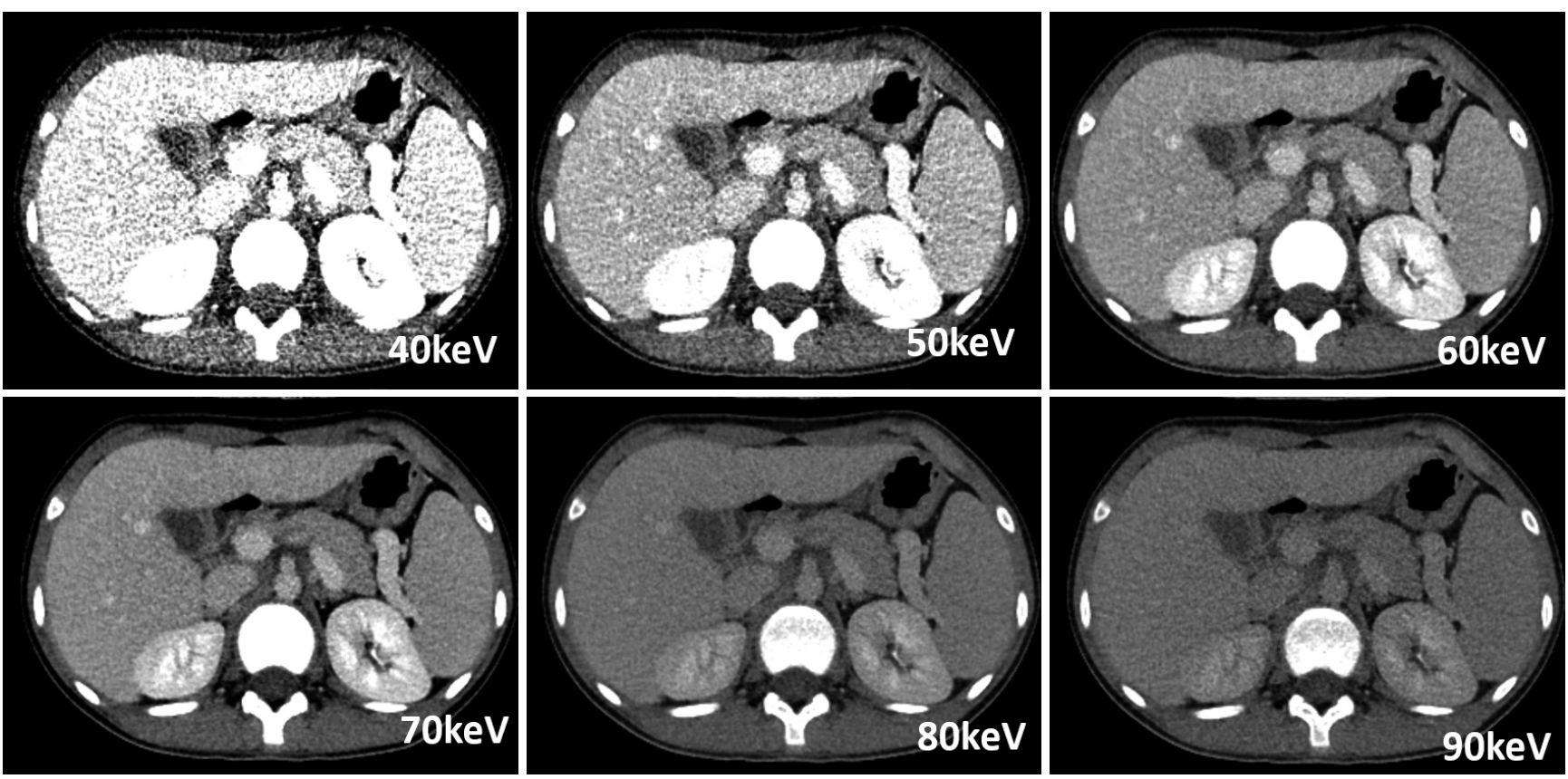

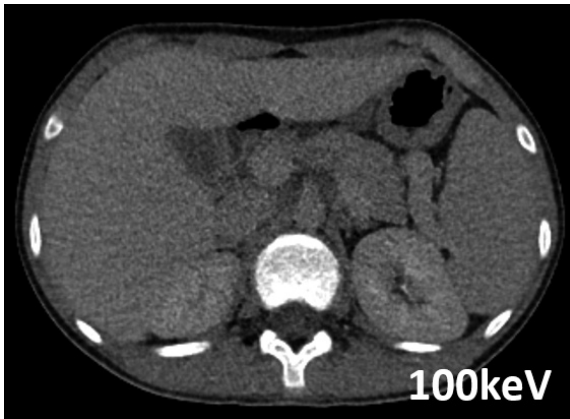

(7). Schabel et al. (23) reported optimal CNR of intrahepatic veins in a poor contrast-enhanced condition obtained at 40 $\mathrm{keV}$ using the noise-optimized VMI technique on late-phase DECT imaging of the upper abdomen in adults. Albrecht et al. (10) reported that CNR at $40 \mathrm{keV}$ achieved peak CNR in DECT angiography of the abdomen in adult population. Our study was in concord with these studies, with all measured organs showing peak CNR at $40 \mathrm{keV}$, gradually decreasing with increasing keV levels, suggesting that noise-optimized VMI can partially overcome noise limitation at low keV levels as shown in the conventional VMI technique. However, we have to be careful, as increased CNR does not always assure the improved image quality.

In adult studies, the effect of noise-optimized algorithm compared with conventional algorithm has been well established. Meier et al. (9) reported that noise-optimized VMI at $40 \mathrm{keV}$ improved the contrast of DECT pulmonary angiography compared to conventional VMI at $60 \mathrm{keV}$. Similarly to previous adult studies, in our study, noiseoptimized VMI at $40 \mathrm{keV}$ showed superior CNR compared to all energy levels in conventional VMI. Compared with the best CNR achieved at $60-70 \mathrm{keV}$ in conventional VMI, the CNR of noise-optimized VMI at $40 \mathrm{keV}$ increased by an average of $285 \%$ in all measured organs. These results may potentially lead to reduction in contrast material with application of noise-optimized algorithm $(3,4)$.

There are not many papers on radiation exposure of DECT in the pediatric population. Goo (24) reported that radiation exposure for dual-source DECT lung perfusion examinations was similar to that found in previous singleenergy CT pulmonary angiogram study performed in children. Recently Zhu et al. (11) indicated that dual-source DECT is dose-neutral in imaging the head and abdomen in children, showing no difference in image quality. In this study, the average CTDIvol of dual-source dual-energy abdominopelvic CT was $3.09 \mathrm{mGy}$, which is lower to that of single-energy abdominopelvic CT (5.05 mGy) reported in a recent pediatric study (12). However, we also have to be aware that other DECT techniques such as rapid voltageswitching method or sequential dual scanning may increase 
Table 6. Comparison of Objective Image Quality Indices between VMIs at $60 \mathrm{keV}$ for Noise-Optimized Algorithm and VMIs at 70 $\mathrm{keV}$ for Conventional Algorithm in Three Measured Organs

\begin{tabular}{|c|c|c|c|c|}
\hline & \multicolumn{2}{|c|}{ Values } & \multicolumn{2}{|c|}{ Relative Difference (\%) } \\
\hline & $\begin{array}{c}\text { Noise-Optimized VMI } \\
\text { at } 60 \mathrm{keV}\end{array}$ & $\begin{array}{c}\text { Conventional VMI } \\
\text { at } 70 \mathrm{keV}\end{array}$ & $\begin{array}{l}\text { Noise-Optimized VMI vs. } \\
\text { Conventional VMI }\end{array}$ & $P$ \\
\hline \multicolumn{5}{|l|}{ Liver } \\
\hline Mean HU & $155.52 \pm 21.44$ & $128.98 \pm 16.57$ & $20.59 \pm 4.41$ & $<0.001^{*}$ \\
\hline SNR & $23.60 \pm 3.30$ & $19.57 \pm 2.51$ & $20.59 \pm 4.50$ & $<0.001^{*}$ \\
\hline CNR & $10.92 \pm 3.51$ & $8.12 \pm 2.64$ & $34.48 \pm 12.44$ & $<0.001^{*}$ \\
\hline \multicolumn{5}{|l|}{ Pancreas } \\
\hline Mean HU & $134.83 \pm 16.89$ & $109.77 \pm 12.07$ & $23.00 \pm 4.92$ & $<0.001^{*}$ \\
\hline SNR & $20.46 \pm 2.60$ & $16.66 \pm 1.83$ & $22.87 \pm 4.98$ & $<0.001^{*}$ \\
\hline CNR & $7.79 \pm 3.10$ & $5.21 \pm 2.23$ & $49.52 \pm 18.62$ & $<0.001^{*}$ \\
\hline \multicolumn{5}{|l|}{ Aorta } \\
\hline Mean HU & $228.69 \pm 27.13$ & $175.64 \pm 20.81$ & $30.50 \pm 4.24$ & $<0.001^{*}$ \\
\hline SNR & $34.71 \pm 4.17$ & $26.65 \pm 3.16$ & $30.24 \pm 4.24$ & $<0.001^{*}$ \\
\hline CNR & $22.03 \pm 4.64$ & $15.20 \pm 3.48$ & $44.93 \pm 8.22$ & $<0.001^{*}$ \\
\hline \multicolumn{5}{|l|}{ Air } \\
\hline Noise & $6.59 \pm 0.76$ & $6.59 \pm 0.85$ & $0.0002 \pm 0.02$ & 0.982 \\
\hline
\end{tabular}

Values are expressed as mean \pm SD. Differences among datasets are expressed by relative difference between noise-optimized and conventional VMIs in percentages and significance $\left({ }^{*}\right)$ is expressed by $p$ value $\leq 0.05$ on basis of paired $t$ test.

radiation exposure and should be used cautiously especially in pediatric patients. Enhanced radiation dose efficiency of the dual-source dual-energy technique coupled with the highest dual-energy spectral contrast additionally available in newer generation dual-source CT system seems to be particularly useful for evaluating children (1).

In this study, we were able to perform dual-source DECT without increasing radiation exposure in pediatric population. Our study demonstrated that noise-optimized VMI technique could increase CNR, SNR, and subjective image quality, compared to the conventional VMI in dualenergy abdominopelvic $\mathrm{CT}$ of children. Especially considering the same effect on the younger group (age 0-10) in subgroup analysis, improved image quality at low keV levels in noise-optimized VMI may enhance diagnostic confidence in small-sized pediatric patients.

Our study has several limitations. Firstly, this study was performed retrospectively, so that selection bias could be occurred. Secondly, this study was a single-center study, and the number of patients was small considering the diversity in body size and composition in children. Although noise-optimized VMI at $60 \mathrm{keV}$ generally showed the best imaging quality of children of our study, some young children may show better image quality at different levels of VMI (e.g., $40 \mathrm{keV}$ or $50 \mathrm{keV}$ ). Therefore, further evaluation may be required in a large study population to present body size-adapted optimal levels covering different age groups in children. Thirdly, this study did not evaluate lesion detectability because of little lesions in the organs of included patients. Future studies will be needed to show noise-optimized algorithm at optimal VMI energy level actually increases the lesion conspicuity in the pediatric abdominopelvic CT.

In conclusion, noise-optimized algorithm shows superior qualitative and quantitative image qualities compared to those of conventional algorithm in dual-energy pediatric abdominopelvic CT. Therefore, noise-optimized algorithm should be applied in pediatric abdominal CT imaging, if available. For overall image quality improvement, $60 \mathrm{keV}$ is recommended in noise-optimized VMI.

\section{Supplementary Materials}

The online-only Data Supplement is available with this article at https://doi.org/10.3348/kjr.2017.0507.

\section{Conflicts of Interest}

Author Seong Yong Pak and Bernhard Krauss are employees of Siemens Healthineers Ltd. However, control of all data and information submitted for publication was given to the authors who were not affiliated with Siemens Healthineers Ltd. For the remaining authors, they have nothing to disclose. 
ORCID

Young Hun Choi

https://orcid.org/0000-0002-1842-9062

Taek Min Kim

https://orcid.org/0000-0001-6915-8795

\section{REFERENCES}

1. Goo HW, Goo JM. Dual-energy CT: new horizon in medical imaging. Korean J Radiol 2017;18:555-569

2. Yu L, Leng S, McCollough CH. Dual-energy CT-based monochromatic imaging. AJR Am J Roentgenol 2012;199(5 Suppl):S9-S15

3. Apfaltrer P, Sudarski S, Schneider D, Nance JW Jr, Haubenreisser $\mathrm{H}$, Fink $\mathrm{C}$, et al. Value of monoenergetic lowkV dual energy CT datasets for improved image quality of CT pulmonary angiography. Eur J Radiol 2014;83:322-328

4. Schneider D, Apfaltrer P, Sudarski S, Nance JW Jr, Haubenreisser $\mathrm{H}$, Fink $\mathrm{C}$, et al. Optimization of kiloelectron volt settings in cerebral and cervical dual-energy CT angiography determined with virtual monoenergetic imaging. Acad Radiol 2014;21:431-436

5. Bamberg F, Dierks A, Nikolaou K, Reiser MF, Becker CR, Johnson TR. Metal artifact reduction by dual energy computed tomography using monoenergetic extrapolation. Eur Radiol 2011;21:1424-1429

6. Leschka S, Stolzmann P, Schmid FT, Scheffel H, Stinn B, Marincek B, et al. Low kilovoltage cardiac dual-source CT: attenuation, noise, and radiation dose. Eur Radiol 2008;18:1809-1817

7. Grant KL, Flohr TG, Krauss B, Sedlmair M, Thomas C, Schmidt B. Assessment of an advanced image-based technique to calculate virtual monoenergetic computed tomographic images from a dual-energy examination to improve contrastto-noise ratio in examinations using iodinated contrast media. Invest Radiol 2014;49:586-592

8. Albrecht MH, Scholtz JE, Kraft J, Bauer RW, Kaup M, Dewes $P$, et al. Assessment of an advanced monoenergetic reconstruction technique in dual-energy computed tomography of head and neck cancer. Eur Radiol 2015;25:2493-2501

9. Meier A, Wurnig M, Desbiolles L, Leschka S, Frauenfelder T, Alkadhi $\mathrm{H}$. Advanced virtual monoenergetic images: improving the contrast of dual-energy CT pulmonary angiography. Clin Radiol 2015;70:1244-1251

10. Albrecht MH, Scholtz JE, Hüsers K, Beeres M, Bucher AM, Kaup $M$, et al. Advanced image-based virtual monoenergetic dual-energy CT angiography of the abdomen: optimization of kiloelectron volt settings to improve image contrast. Eur Radiol 2016;26:1863-1870

11. Zhu X, McCullough WP, Mecca P, Servaes S, Darge K. Dualenergy compared to single-energy $\mathrm{CT}$ in pediatric imaging: a phantom study for DECT clinical guidance. Pediatr Radiol 2016;46:1671-1679

12. Siegel MJ, Curtis WA, Ramirez-Giraldo JC. Effects of dual- energy technique on radiation exposure and image quality in pediatric body CT. AJR Am J Roentgenol 2016;207:826-835

13. Nievelstein RA, van Dam IM, van der Molen AJ. Multidetector CT in children: current concepts and dose reduction strategies. Pediatr Radiol 2010;40:1324-1344

14. Lubner MG, Pickhardt PJ, Tang J, Chen GH. Reduced image noise at low-dose multidetector CT of the abdomen with prior image constrained compressed sensing algorithm. Radiology 2011;260:248-256

15. Marin D, Nelson RC, Schindera ST, Richard S, Youngblood RS, Yoshizumi TT, et al. Low-tube-voltage, high-tube-current multidetector abdominal CT: improved image quality and decreased radiation dose with adaptive statistical iterative reconstruction algorithm--initial clinical experience. Radiology 2010;254:145-153

16. Winklhofer S, Lambert JW, Sun Y, Wang ZJ, Sun DS, Yeh BM. Pelvic beam-hardening artifacts in dual-energy CT image reconstructions: occurrence and impact on image quality. AJR Am J Roentgenol 2017;208:114-123

17. MacDougall RD, Kleinman PL, Yu L, Lee EY. Pediatric thoracic CT angiography at $70 \mathrm{kV}$ : a phantom study to investigate the effects on image quality and radiation dose. Pediatr Radiol 2016;46:1114-1119

18. Yu L, Bruesewitz MR, Thomas KB, Fletcher JG, Kofler JM, McCollough $\mathrm{CH}$. Optimal tube potential for radiation dose reduction in pediatric CT: principles, clinical implementations, and pitfalls. Radiographics 2011;31:835-848

19. Verdun FR, Lepori D, Monnin P, Valley JF, Schnyder P, Gudinchet F. Management of patient dose and image noise in routine pediatric CT abdominal examinations. Eur Radiol 2004; 14:835-841

20. Sudarski S, Apfaltrer P, Nance JW Jr, Schneider D, Meyer $M$, Schoenberg S0, et al. Optimization of keV-settings in abdominal and lower extremity dual-source dual-energy CT angiography determined with virtual monoenergetic imaging. Eur J Radiol 2013;82:e574-e581

21. Pomerantz SR, Kamalian S, Zhang D, Gupta R, Rapalino 0, Sahani DV, et al. Virtual monochromatic reconstruction of dual-energy unenhanced head CT at 65-75 keV maximizes image quality compared with conventional polychromatic CT. Radiology 2013;266:318-325

22. Matsumoto K, Jinzaki M, Tanami Y, Ueno A, Yamada M, Kuribayashi S. Virtual monochromatic spectral imaging with fast kilovoltage switching: improved image quality as compared with that obtained with conventional 120-kVp CT. Radiology 2011;259:257-262

23. Schabel C, Bongers M, Sedlmair M, Korn A, Grosse U, Mangold $S$, et al. Assessment of the hepatic veins in poor contrast conditions using dual energy CT: evaluation of a novel monoenergetic extrapolation software algorithm. Rofo 2014;186:591-597

24. Goo HW. Initial experience of dual-energy lung perfusion CT using a dual-source CT system in children. Pediatr Radiol 2010;40:1536-1544 